\title{
openheart Risk factors of diabetic cardiac autonomic neuropathy in patients with type 1 diabetes mellitus: a meta-analysis
}

\author{
Mohamed D Dafaalla, ${ }^{1}$ Mohammed N Nimir, ${ }^{2}$ Mosab I Mohammed, ${ }^{2}$ Omer A Ali, ${ }^{2}$ \\ Abbashar Hussein ${ }^{3}$
}

To cite: Dafaalla MD, Nimir MN, Mohammed MI, et al. Risk factors of diabetic cardiac autonomic neuropathy in patients with type 1 diabetes mellitus: a meta-analysis. Open Heart 2016;3:e00336.

doi:10.1136/openhrt-2015000336

Received 12 September 2015 Revised 25 April 2016 Accepted 30 June 2016

\section{CrossMark}

\footnotetext{
${ }^{1}$ Soba Center for Audit and Research (SCAR), University of Khartoum, Khartoum, Sudan

${ }^{2}$ Daoud Research Group, University of Khartoum, Khartoum, Sudan

${ }^{3}$ Faculty of Medicine, Daoud Research Group, University of Khartoum, Khartoum, Sudan

Correspondence to Dr Mohamed D Dafaalla; mdafaallah200@gmail.com
}

\section{ABSTRACT}

Objectives: We aimed to stratify the possible risk factors for diabetic cardiac autonomic neuropathy (CAN).

Methods: We did a meta-analysis of risk factors of CAN. We did a web-based search for literature in MEDLINE/PubMed, Scopus database and CENTRAL database up to August 2015. We included clinical trials or cohort studies that provide data about relationship between CAN and variables of interest. Our risk factors of interest were age, sex, duration of diabetes, body mass index (BMI), systolic blood pressure (sBP) and diastolic blood pressure (dBP), glycated haemoglobin (HbA1C), high-density lipoprotein and low-density lipoprotein (HDL and $L D L)$, triglycerides, retinopathy and nephropathy. We generated Forest plots, $\chi^{2}$ test and $\mathrm{I}^{2}$ as tests for heterogeneity, risk ratio (RR), mean difference (MD), Cls and p values by ReVMan V.5.3 software.

Results: We found a total of 882 related items. We excluded 873 studies from the title and abstract and 4 studies after review of full reports. Four studies were included. Our meta-analysis showed significant association between CAN and age (MD=4.94 (3.46 to $6.42)$ ), duration of diabetes ( $\mathrm{MD}=4.51$ (2.51 to 6.52$)$ ), $\mathrm{HbA1C}(\mathrm{MD}=0.48$ (0.28 to 0.67)), BMI (MD=0.55 (0.08 to 1.01$)$ ), serum triglycerides $(M D=0.09(0.01$ to 0.17$))$, proliferative retinopathy ( $R R=3.69$ (1.20 to 11.34$))$, microalbuminuria ( $R R=2.47$ (1.43 to 4.29)), hypertension ( $R R=4.18$ (2.52 to 6.91$)$ ) and $\mathrm{sBP}$ $(\mathrm{MD}=4.10$ (2.20 to 6.00$))$. We neither discovered the absence of significant association between the development of CAN and male sex ( $R R=1.57$ (0.45 to 5.39)), $\mathrm{dBP}(\mathrm{MD}=0.89$ (-0.36 to 2.14)), cholesterol level ( $M D=1.19(-0.99$ to 3.36$)), L D L(M D=0.12(-0.15$ to 0.39$))$, nor HDL level (MD=-0.28 (-0.58 to 0.03$))$.

Conclusions: Age, duration of diabetes, $\mathrm{HbA1C}, \mathrm{BMI}$, serum triglycerides, proliferative retinopathy, microalbuminuria, hypertension and sBP are directly related to the risk of development of diabetic CAN.

\section{INTRODUCTION}

Diabetes affects more than million worldwide. Diabetic neuropathies, including cardiac autonomic neuropathy (CAN), are a common chronic complication of type 1 and

\section{KEY MESSAGES}

What is already known about this subject?

- Diabetic cardiac autonomic neuropathy (CAN) is a frequent chronic complication of diabetes mellitus with potentially life-threatening outcomes. $65 \%$ with age and diabetes duration. It is also a major source of increased cost in caring for the patient with diabetes.

- Previous studies showed variable risk factors for CAN.

- Currently there is no acceptable tool to stratify risk of development of CAN.

What does this study add?

- This study stratifies the risk factors for CAN.

How might this impact on clinical practice?

- This study may form the foundation for formulation of valid predictor or risk stratification tools for CAN.

type 2 diabetes and confer high morbidity and mortality to patients with diabetes. ${ }^{1}$ Diabetic autonomic neuropathy is among the least recognised and understood complications of diabetes, despite its significant negative impact on survival and quality of life in people with diabetes. ${ }^{2} \mathrm{CAN}$ is the impairment of cardiovascular autonomic control in the setting of diabetes after exclusion of other causes. The prevalence of confirmed CAN is $\sim 20 \%$ and increases up to $65 \%$ with age and diabetes duration. ${ }^{34}$ It is a frequent chronic complication of diabetes mellitus with potentially life-threatening outcomes. ${ }^{5}$ It is also a major source of increased cost in caring for the patient with diabetes. The metabolic disorders of diabetes lead to diffuse and widespread damage of peripheral and autonomic nerves, and small vessels. When diabetic neuropathy affects the autonomic nervous system, it can damage the cardiovascular, gastrointestinal, genitourinary and neurovascular systems and impair metabolic functions such as glucose counter-regulation. ${ }^{6}$ 
CAN is associated with a high risk of cardiac arrhythmias and with sudden death. Longitudinal studies of participants with CAN have shown 5-year mortality rates 16-50\% in type 1 and type 2 diabetes, with a high proportion attributed to sudden cardiac death. ${ }^{7-9}$ In the EURODIAB Prospective Cohort Study of 2787 patients with type 1 diabetes mellitus, CAN was the strongest predictor for mortality during a 7-year follow-up, exceeding the effect of traditional cardiovascular risk factors. ${ }^{10}$ The Hoorn study reported that the presence of diabetic CAN doubled the 9-year mortality risk in an elderly cohort. ${ }^{11}$ A meta-analysis of 15 studies, including 2900 participants with diabetes, reported a pooled relative risk of mortality of 3.45 (95\% CI 2.66 to 4.47 ) in patients with CAN, with a progressive increase in the risk with the increase in the number of abnormal CAN function tests. ${ }^{12}$ Diabetic CAN is eventually caused by complex interactions among a number of pathogenic pathways. Hyperglycaemia is the leading cause of the initiation of this pathogenic process. ${ }^{2} 13$

There is no widely accepted single approach to the diagnosis of CAN in diabetes. Assessment of HRV, orthostatic hypotension and 24-h blood pressure profiles provides indexes of parasympathetic and sympathetic autonomic functions and can be used in clinical settings. Other methods, such as cardiac sympathetic imaging, microneurography, occlusion plethysmography and baroreflex sensitivity, are currently used predominantly in research settings, but may find a place in the clinical assessment of CAN in the future. ${ }^{1}$ In practice, criteria for CAN diagnosis and staging are: (1) one abnormal cardiovagal test result identifies possible or early CAN; (2) at least two abnormal cardiovagal test results are required for definite or confirmed CAN and (3) the presence of orthostatic hypotension in addition to abnormal heart rate test results identifies severe or advanced CAN. Improving glycaemic control, lifestyle changes and cardiovascular disease risk factors management are the mainstay of treatment, which generally slow the progression of CAN rather than reversing it. Disease-modifying treatment is lacking. ${ }^{14}$

We aimed to stratify risk factors for diabetic CAN by evaluating possible association between CAN and the risk factors, including age, sex, duration of diabetes (years), body mass index (BMI $\left.\left(\mathrm{kg} / \mathrm{m}^{2}\right)\right)$, systolic blood pressure $(\mathrm{sBP})$ and diastolic blood pressure $(\mathrm{dBP}(\mathrm{mm}$ $\mathrm{Hg})$ ), glycated haemoglobin (HbAlc), high-density lipoprotein and low-density lipoprotein (HDL and LDL), triglycerides and the presence of other complications as retinopathy and nephropathy.

\section{METHODS}

\section{Sources}

We did a web-based search for literature in MEDLINE/ PubMed, Scopus database and Cochrane Central Register of Controlled Trials databases up to August 2015. We used a combination of keywords 'type 1 diabetes' and 'CAN' for search.
We got unpublished study ${ }^{15}$ by help of a senior consultant. Two authors, MDD and MNN, extracted data separately and matched their results. We included randomised controlled trials and cohort studies that provide some or all data about relationship between CAN and variables of interest in the last 15 years. The search strategy had no language restrictions. We assessed the relevance of studies by using a hierarchical approach based on title, abstract and the full manuscript. We also consulted references from the extracted articles and reviews to complete the data bank. We assessed the relevance of studies by using a hierarchical approach based on title, abstract and the full manuscript. The study population of included studies should be the diabetic patients with type 1 diabetes mellitus (IDDM). We excluded studies that studied CAN in non-diabetic patients or did not include data about values of variables of interest.

\section{Study selection}

In analysis of association of each variable versus diabetic CAN, we included only studies that provide the mean, SD, total population of participants with and without diabetic CAN if variable data are continuous and studies that provide the variable number/proportion of participants and total population of participants with and without diabetic CAN if variable data are dichotomous. The quality of studies was evaluated using the GRADE approach. The GRADE approach defines the quality of a body of evidence as the extent to which one can be confident that an estimate of effect or association is close to

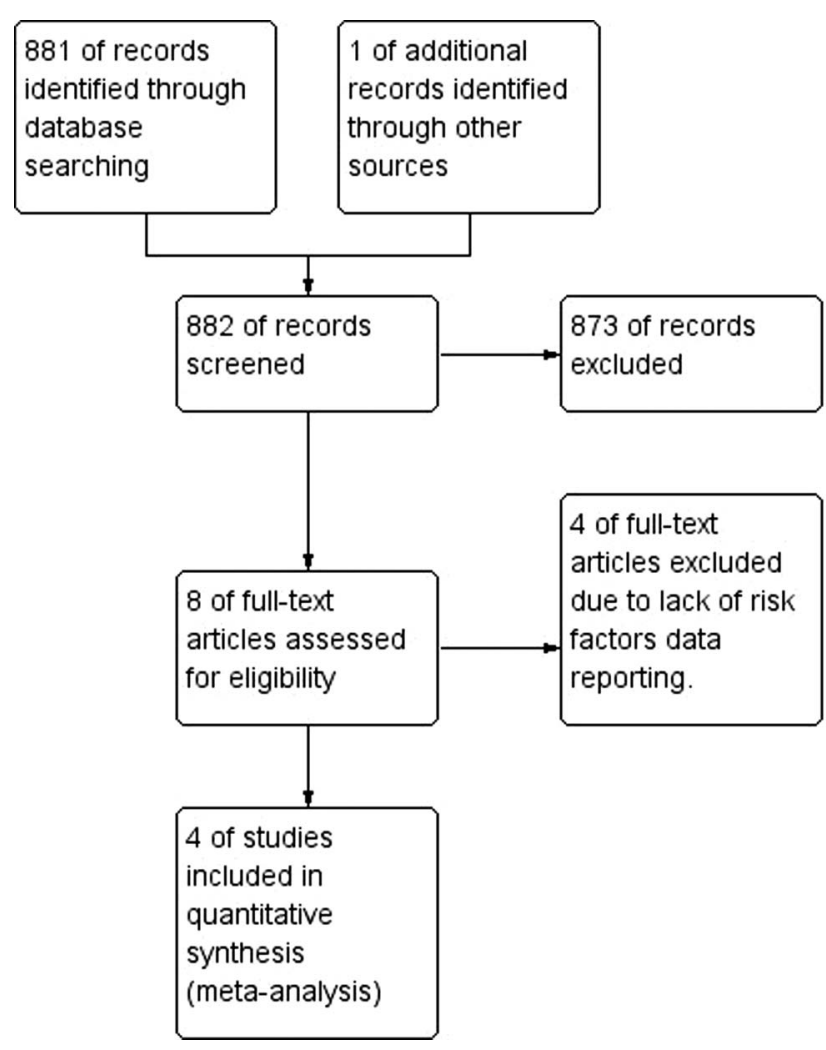

Figure 1 PRISMA flow chart of study selection process. 
Table 1 Characteristics of included studies

\begin{tabular}{|c|c|c|c|c|c|c|}
\hline Author, year & Journal & $\begin{array}{l}\text { Source of study } \\
\text { population }\end{array}$ & Method for diagnosing CAN & Inclusion criteria & $\begin{array}{l}\text { Number of } \\
\text { participants }\end{array}$ & $\begin{array}{l}\text { Mean } \\
\text { follow-up } \\
\text { duration } \\
\text { (years) }\end{array}$ \\
\hline Stella $2000^{3}$ & $\begin{array}{l}\text { Journal of } \\
\text { Diabetes and its } \\
\text { Complications }\end{array}$ & $\begin{array}{l}\text { Pittsburgh } \\
\text { Epidemiology of } \\
\text { Diabetes } \\
\text { Complications } \\
\text { Prospective Study } \\
\text { (EDC Study) }\end{array}$ & $\begin{array}{l}\text { Heart rate response to deep } \\
\text { breathing }\end{array}$ & Patients with type 1 diabetes & 373 & 4.7 \\
\hline Witte $2005^{23}$ & Diabetologia & $\begin{array}{l}\text { Part of the } \\
\text { EURODIAB } \\
\text { Prospective } \\
\text { Complications Study }\end{array}$ & $\begin{array}{l}\text { Loss of heart rate variability or } \\
\text { postural hypotension on standing }\end{array}$ & Patients with type 1 diabetes & 956 & 7.3 \\
\hline Mogenson $2012^{21}$ & Diabetes & $\begin{array}{l}\text { Type } 1 \text { diabetic } \\
\text { patients registry }\end{array}$ & $\begin{array}{l}\text { CAN was defined as two or more } \\
\text { abnormal tests: heart rate } \\
\text { variability during deep breathing, } \\
\text { Valsalva ratio, lying-to-standing } \\
\text { test and blood pressure } \\
\text { response to standing up }\end{array}$ & $\begin{array}{l}\text { Inclusion criteria were type } 1 \\
\text { diabetes according to American } \\
\text { Diabetes Association criteria of at } \\
\text { least } 10 \text { years' duration, age } \\
\text { between } 18 \text { and } 75 \text { years and } \\
\mathrm{HbA} 1 \mathrm{C}, 10 \% \text {. Exclusion criteria } \\
\text { were albuminuria (urinary } \\
\text { albumin-to-creatinine ratio } 30 \mathrm{mg} / \mathrm{g} \text {; } \\
\text { elevated S-creatinine } 120 \mathrm{mmol} / \mathrm{L}) \text {, } \\
\text { untreated hypertension }(0.140 / \\
85 \mathrm{~mm} \mathrm{Hg} \text { ) and electrocardiographic } \\
\text { signs or clinical symptoms of heart } \\
\text { disease }\end{array}$ & 56 & Not reported \\
\hline Orlov $2015^{22}$ & $\begin{array}{l}\text { Clinical Journal } \\
\text { of American } \\
\text { Society of } \\
\text { Nephrology }\end{array}$ & $\begin{array}{l}\text { The First Joslin } \\
\text { Kidney Study }\end{array}$ & Heart rate variability & $\begin{array}{l}\text { Patients with type } 1 \text { diabetes at the } \\
\text { Joslin Diabetes Center }\end{array}$ & 370 & 14 \\
\hline
\end{tabular}


the quantity of specific interest. Quality of a body of evidence involves consideration of within-study risk of bias (methodological quality), directness of evidence, heterogeneity, precision of effect estimates and risk of publication bias. The GRADE system entails an assessment of the quality of a body of evidence for each individual outcome. The GRADE approach specifies four levels of quality. The highest quality rating is for randomised trial evidence. Review authors will generally grade evidence from sound observational studies as low quality. If, however, such studies yield large effects and there is no obvious bias explaining those effects, review authors may rate the evidence as moderate or-if the effect is large enough-even high quality. The very low-quality level includes, but is not limited to, studies with critical problems and unsystematic clinical observations (eg, case series or case reports). ${ }^{16}$

We extracted data from the original reports by using a standardised data extraction form. Two investigators (MDD and MNN) collected the data, and disagreements were solved by consensus and by the opinion of a third author (OAA), if necessary. We extracted the following baseline characteristics from the original reports by using a standardised data extraction form and included them in the meta-analysis: lead author, year of publication, study design, participants and outcomes assessed. Our outcomes of interest we included them in the meta-analysis are: age, sex, duration of diabetes (years), BMI $\left(\mathrm{kg} / \mathrm{m}^{2}\right)$, sBP and dBP $(\mathrm{mm} \mathrm{Hg})$, glycated haemoglobin (HbA1c), high-density lipoprotein and lowdensity lipoprotein (HDL and LDL), triglycerides and the presence of other complications as retinopathy and nephropathy. We used the Cochrane Collaboration's tool for assessing risk of bias to evaluate bias in included studies. It is a two-part tool, addressing the seven specific domains (namely sequence generation, allocation concealment, blinding of participants and personnel, blinding of outcome assessment, incomplete outcome data, selective outcome reporting and 'other issues'). Each domain in the tool includes one or more specific entries in a 'risk of bias' table. Within each entry, the first part of the tool describes what was reported to have happened in the study, in sufficient detail to support a judgement about the risk of bias. The second part of the tool assigns a judgement relating to the risk of bias for that entry. This is achieved by assigning a judgement of 'Low risk' of bias, 'High risk' of bias or 'Unclear risk' of bias. We intended to assess publication bias using funnel plot techniques, Begg's rank test and Egger regression test as appropriate given the known limitations of these methods. We used RevMan V.5.3 for Windows by the Cochrane Collaboration to analyse data. For continuous variables, we used the mean difference (MD) as effect measure and calculated summary relative risks with $95 \%$ CIs by using an inverse variance method. For dichotomous data, we used risk ratio, OR and/or risk difference as effect measure. Following data entry, we generated Forest plots, $\chi^{2}$ test and $\mathrm{I}^{2}$ as tests for heterogeneity and

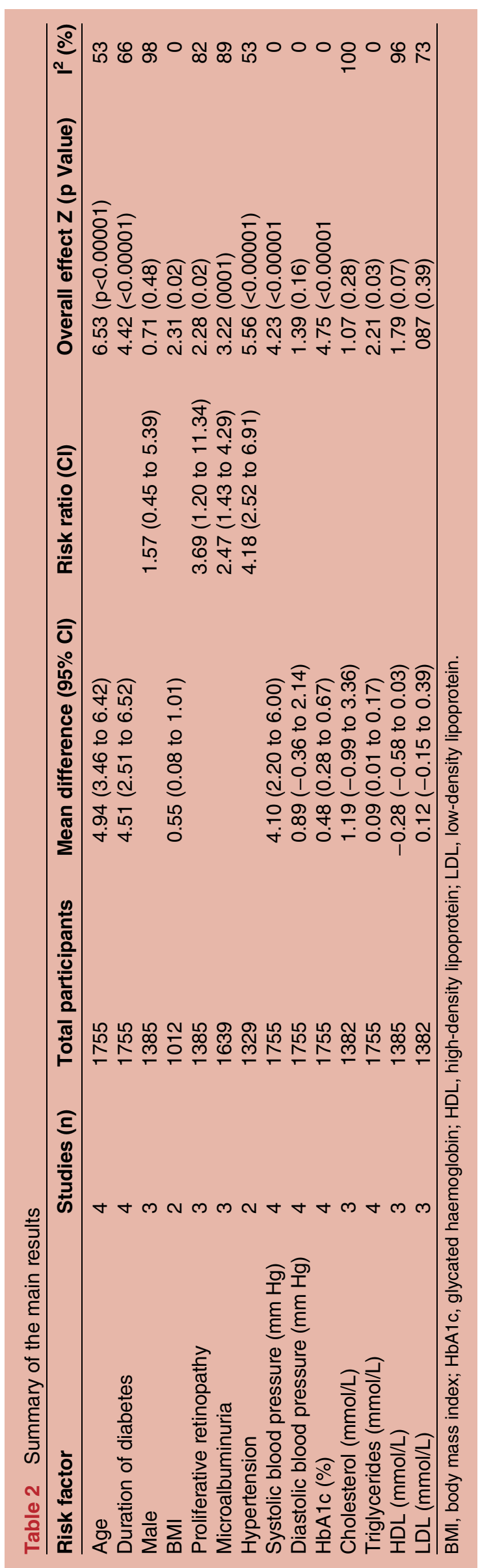


$\mathrm{p}$ values by ReVMan V.5.3. We used the fixed-effects model when we found out $\mathrm{I}^{2}<25 \%$; otherwise, we implemented the random-effects model. We calculated the number of participants for participants diagnosed with (+ve) CAN and (-ve) CAN from studies provided ratios from total population.

\section{RESULTS}

We found a total of 882 related items: one of them was unpublished report. We excluded 873 studies from the title and abstract. We excluded additional four studies referenced $^{17-20}$ after review of full reports because the presentation of data in reports does not allow differentiation between the values of outcomes of interest in patients with and without CAN separately (figure 1) shows the process and results of study selection.

We identified four studies referenced ${ }^{321-23}$ compatible with our eligibility criteria and provide comprehensive data of clinical parameters that could be risk factors for CAN. One study was performed as a cohort study as a part of clinical trials, ${ }^{23}$ whereas the remaining three were cohort studies. ${ }^{3}{ }^{21-23}$ A study by Witte (2005) was implemented as part of the EURODIAB Prospective Complications Study. Stella (2000) was performed in participants from the
Epidemiology of Diabetes Complications Study. A study by Mogenson (2012) was established on patients who were recruited from the outpatient clinic cohort of patients with type 1 diabetes mellitus at Steno Diabetes Center and the Diabetes Unit, Rigshospitalet. A study by Orlov (2015) was performed in patients from the First Joslin Kidney Study. Table 1 illustrates the major details of included studies.

All included studies used heart rate variability as a diagnostic tool, and some used additional tests. Since there were no control groups involved in the included studies and all individuals under study were patients with type 1 diabetes, the risk of performance bias due to improper blinding of patients or variables assessment was considered low. Attrition bias was considered low because of the absence of missing data. We could not use funnel plot techniques, Begg's rank test and Egger regression test because the number of included studies is small. Rosenthal's basic Fail-Safe Number was 16.2. In other words, we need $>16$ studies not included in this meta-analysis to make the $p$ value insignificant. This indicates that probability of publication bias is low.

We considered the selection bias in Stella 2000, Witte 2005 and Orlove 2015 low because included participants were recruited from the Pittsburgh Epidemiology of

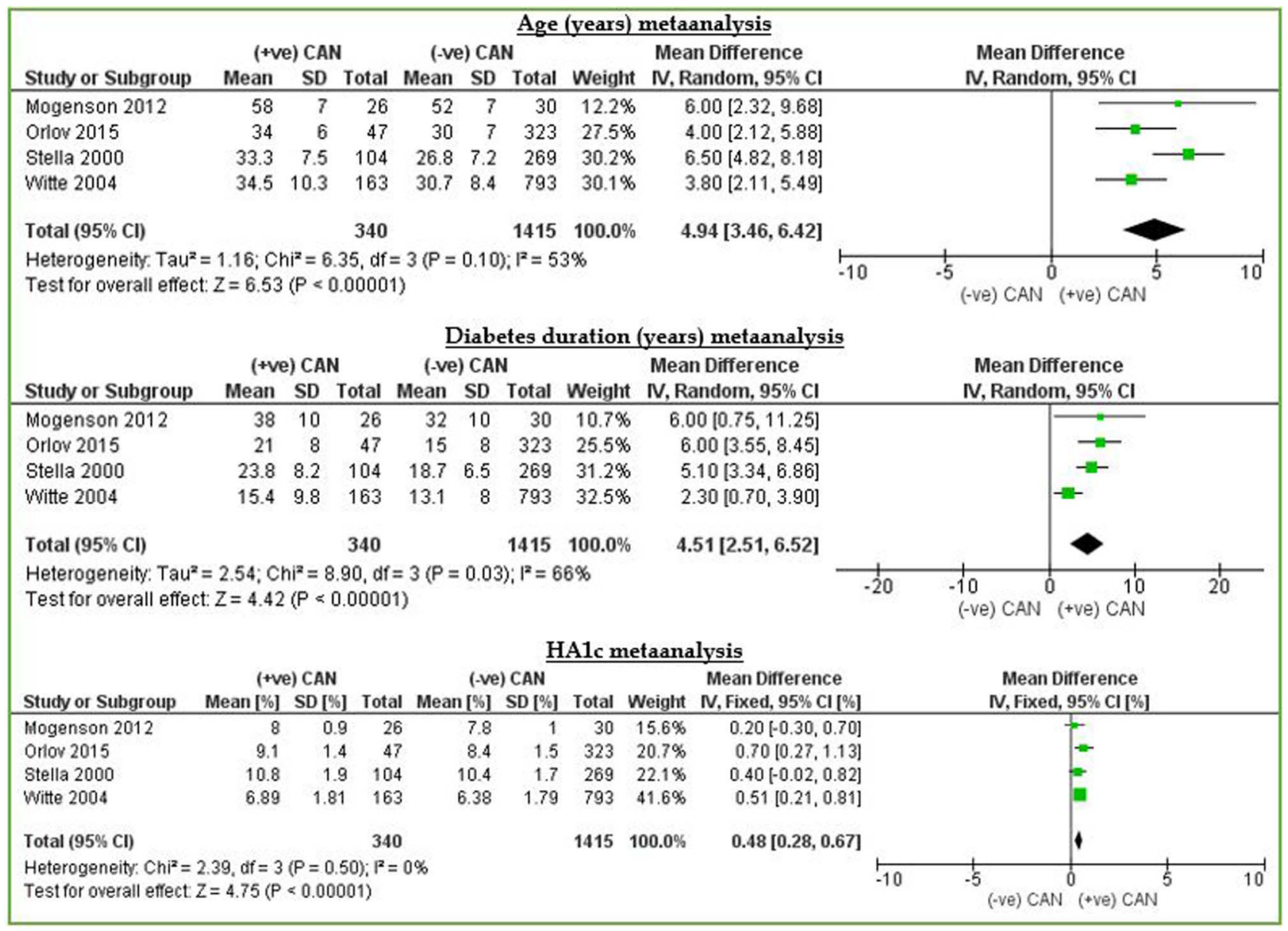

Figure 2 CAN versus age, duration of diabetes and HbA1c. CAN, cardiac autonomic neuropathy; HbA1c, glycated haemoglobin. 


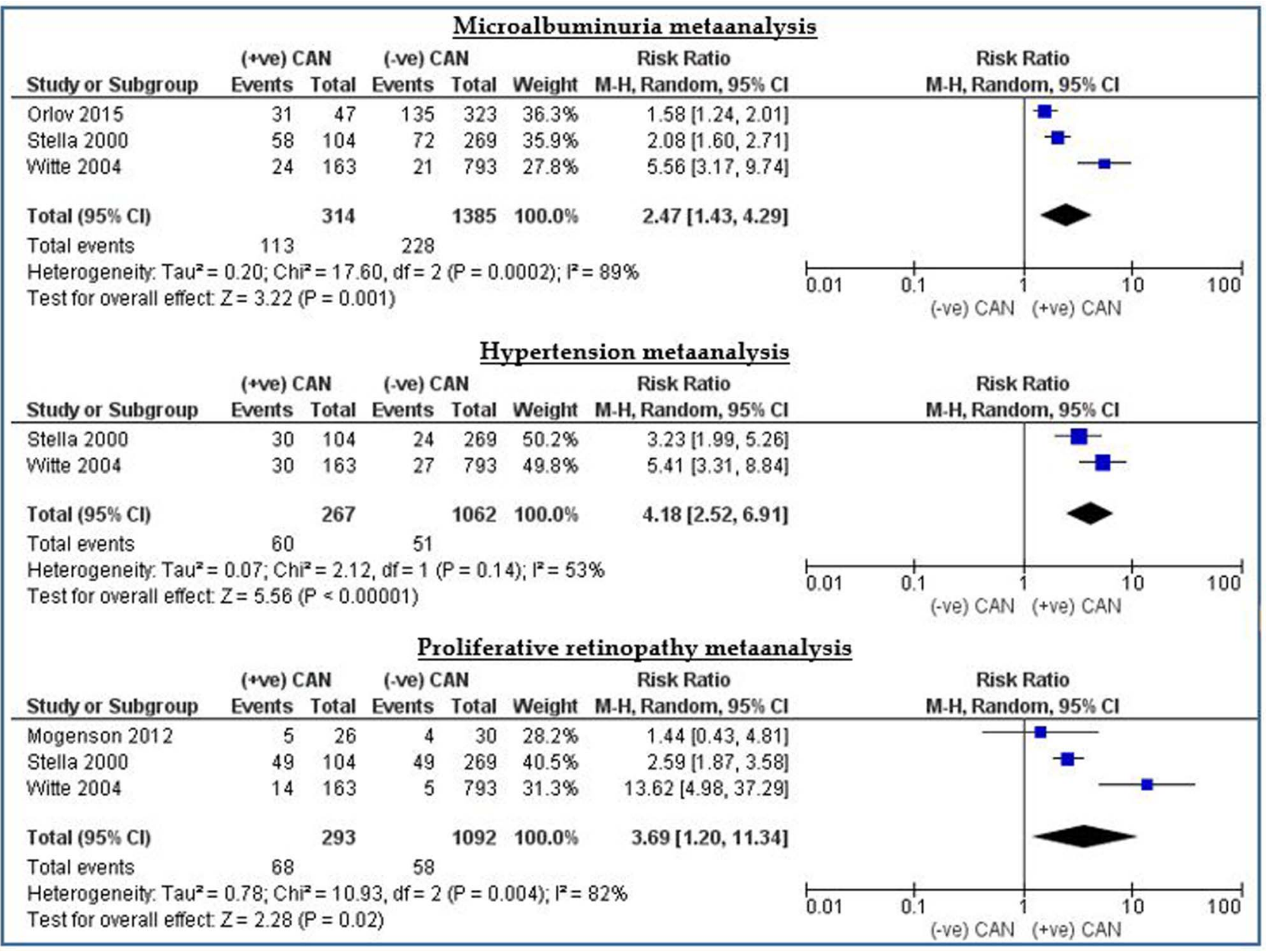

Figure 3 CAN versus hypertension, microalbuminuria and proliferative retinopathy. CAN, cardiac autonomic neuropathy.

Diabetes Complications Study, the EUROBIAB IDDM Complications Study and the Joslin Study of the Natural History of Microalbuminuria, respectively, where participants were enrolled randomly. However, the risk of bias in Mogenson 2012 was unclear because authors did not report a selection method. Yet, there was no serious issue to downgrade the quality of information reported in the study.

Our meta-analysis showed significant statistical association between CAN and the following risk factors: age, duration of diabetes, HbA1c, BMI, serum triglycerides, proliferative retinopathy, microalbuminuria, hypertension and sBP. We neither discovered the absence of significant association between the development of CAN and male sex, dBP, cholesterol level, LDL, nor HDL level. Table 2 summarises the main results of this meta-analysis.

\section{DISCUSSION}

\section{Summary of findings}

Regarding age and duration of diabetes, analysis revealed a significant difference in age and duration of diabetes between patients diagnosed with diabetic CAN and patients without diabetic CAN with an MD of $>4$ years and a half in favour of patients with CAN. Likewise, there was statistically significant association between HbA1c and the development of diabetic CAN. The MD between levels of $\mathrm{HbAlc}$ was $0.48 \%$ in favour of patients diagnosed with diabetic CAN. This indicates that the longer the period of illness and the higher levels of $\mathrm{HgAlc}$, the higher risk of developing CAN is present. This is consistent with the hypothesis that impaired glucose control is responsible for the pathogenesis of diabetic CAN over the years, which means that diabetes duration and control are important factors in determining the possibility of developing CAN. In contrary, according to table 2, the risk of developing CAN appears to be same in males and females (figure 2).

The analysis showed significant relationships between diabetic CAN and complications of diabetes, namely microalbuminuria, proliferative retinopathy and hypertension with $\mathrm{p}$ values of $<0.005$ for all, and relative risks of 2.96, 3.69 and 4.19, respectively. In other words, patients with diabetes who develop microalbuminuria or proliferative retinopathy have a threefold increased risk of getting diabetic CAN compared to those with diabetes who are free from these complications. The risk will rise 


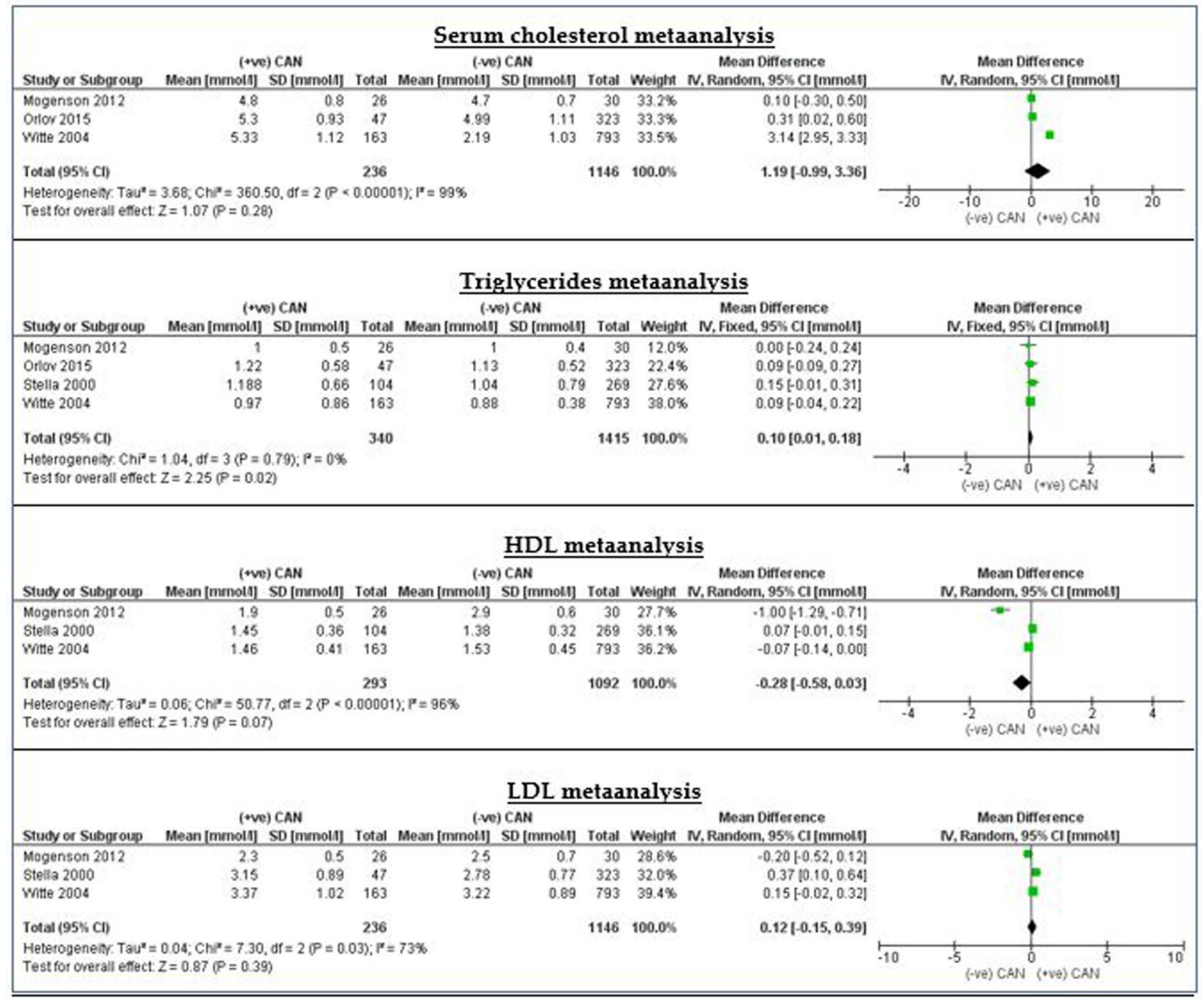

Figure 4 Meta-analysis of the HDL, LDL and triglycerides. HDL, high-density lipoprotein; LDL, low-density lipoprotein.

to reach fourfolds if the patient is hypertensive. Figure 3 illustrates this result.

Regarding the relationship with the blood pressure, analysis illustrated strong association between $\mathrm{sBP}$ and diabetic CAN ( $\mathrm{p}=0.005)$ with an $\mathrm{MD}$ of $4.1 \mathrm{~mm} \mathrm{Hg}$ in favour of patients diagnosed with diabetic CAN. On the other hand, there was no significant difference in $\mathrm{dBP}$ between patients with and without CAN with MD of $<1 \mathrm{~mm} \mathrm{Hg}$.

Results demonstrated weak relationship between CAN and lipid profile parameters. The CIs of serum cholesterol, LDL and HDL cross the line of no effect, indicating the absence of significant association between them and the development of CAN. However, serum triglyceride level and BMI have a significant direct relationship with CAN in patients with diabetes $(p=0.02$ for both). Nevertheless, the overall effect estimate was away from the line of no effect by only $0.09 \mathrm{~mol} / \mathrm{L}$ and $0.08 \mathrm{~kg} / \mathrm{m}^{2}$ consecutively. This indicates that this association seems to be weak and most likely will be changed significantly by future research. In short, abnormal lipid profile is not a reliable risk factor for CAN in patients with diabetes (figure 4).

The role of intensive glycaemic control in prevention of CAN was confirmed by clinical trials. ${ }^{18}$ However, when we compared the strong results deduced from data of diabetes complication and compared them with indicators of glycaemic control (like HA1c and lipid profile) whose association with CAN was weaker, we come out with two possible theories. The first is that the loss of glycaemic control is a common risk factor for CAN and other complications, and the association detected is a mirror of concomitant occurrence of CAN with other diabetes complications. This theory is augmented by the fact that some researches highlighted the CAN as an independent risk factor for renal function decline in patients with type 2 diabetes. ${ }^{4}$ The second theory states the nephropathy, retinopathy and hypertension as risk factors that precede the occurrence of CAN, and since the main protective method against these complications is to control blood sugar, it is likely to find out strong association between CAN and glycaemic control. In both cases, intensive 
glycaemic control appears to be the most efficient way to prevent CAN, but identification of the exact pathogenesis and relationship between CAN and other diabetes complications may give opportunity to develop new therapeutic strategies to make earlier efficient intervention, especially in patients with advanced disease or difficult to control blood glucose levels.

\section{Quality of the evidence}

The other important element in the grade system we must consider is the heterogeneity. We downgraded the quality of meta-analysis of risk factors that showed $\mathrm{I}^{2}$ score $>75 \%$ (ie, severe heterogeneity). Since the risk of bias is low and there are no serious factors to downgrade evidence from included studies, we considered the results of analysis of male gender, proliferative retinopathy, microalbuminuria, HDL, LDL and cholesterol as moderate quality evidence due to unexplained heterogeneity. We consider the remaining results as high-quality results.

\section{Implications for practice}

This meta-analysis enables identification of patients at high risk of getting diabetic CAN. This may allow earlier detection and help provide appropriate management at early stages of disease. Moreover, this will provide more time to vigorously manage diabetes in order to prevent CAN. Furthermore, this will increase the efficacy of interventions that may fail at advanced diabetic CAN. This will improve the survival, quality of life and life expectancy among diabetic patients.

\section{Implications for research}

This study opens the doors for further research in aspects related to the risk factors and predictors of diabetic CAN to consolidate evidence from previous research. It may also form the base for the development of clinical score to stratify the risk of developing CAN in patients with type 1 diabetes. The process of score formulation, testing and validation is considered a rich resource of high-quality ideas for researchers.

\section{Limitations}

The study has some limitations to consider. There are relatively few studies fulfilled the meta-analysis criteria and the small number of participants with CAN. This indicates the need for more studies, especially clinical trials, which focus on CAN.

Twitter Follow Musab Mohammed at @ganas90

Contributors MDD and OAA performed formulation of idea. MDD and MNM conducted data extraction and analysis. MIM and MDD helped in writing the manuscript. AH performed formulation of idea.

Competing interests None declared.

Provenance and peer review Not commissioned; externally peer reviewed.

Data sharing statement No additional data are available.

Open Access This is an Open Access article distributed in accordance with the Creative Commons Attribution Non Commercial (CC BY-NC 4.0) license, which permits others to distribute, remix, adapt, build upon this work non- commercially, and license their derivative works on different terms, provided the original work is properly cited and the use is non-commercial. See: http:// creativecommons.org/licenses/by-nc/4.0/

\section{REFERENCES}

1. Michel-Chavez A, Estanol B, Gien-Lopez JA, et al. Heart rate and systolic blood pressure variability on recently diagnosed diabetics. Arq Bras Cardiol 2015;105:276-84.

2. Pop-Busui R. Cardiac autonomic neuropathy in diabetes: a clinical perspective. Diabetes Care 2010;33:434-41.

3. Stella $P$, Ellis D, Maser RE, et al. Cardiovascular autonomic neuropathy (expiration and inspiration ratio) in type 1 diabetes. Incidence and predictors. J Diabetes Complicat 2000;14:1-6.

4. Tahrani AA, Dubb K, Raymond NT, et al. Cardiac autonomic neuropathy predicts renal function decline in patients with type 2 diabetes: a cohort study. Diabetologia 2014;57:1249-56.

5. Balcioglu AS, Muderrisoglu H. Diabetes and cardiac autonomic neuropathy: clinical manifestations, cardiovascular consequences, diagnosis and treatment. World J Diabetes 2015;6:80-91.

6. Vinik Al, Erbas T, Casellini CM. Diabetic cardiac autonomic neuropathy, inflammation and cardiovascular disease. J Diabetes Investig 2013;4:4-18.

7. Ewing DJ, Campbell IW, Clarke BF. Assessment of cardiovascular effects in diabetic autonomic neuropathy and prognostic implications. Ann Intern Med 1980;92(2 Pt 2):308-11.

8. Navarro X, Kennedy WR, Sutherland DE. Autonomic neuropathy and survival in diabetes mellitus: effects of pancreas transplantation. Diabetologia 1991;34(Suppl 1):S108-12.

9. O'Brien IA, McFadden JP, Corrall RJ. The influence of autonomic neuropathy on mortality in insulin-dependent diabetes. $Q \mathrm{~J}$ Med 1991;79:495-502.

10. Soedamah-Muthu SS, Chaturvedi N, Witte DR, et al. Relationship between risk factors and mortality in type 1 diabetic patients in Europe: the EURODIAB Prospective Complications Study (PCS). Diabetes Care 2008;31:1360-6.

11. Gerritsen J, Dekker JM, TenVoorde BJ, et al. Impaired autonomic function is associated with increased mortality, especially in subjects with diabetes, hypertension, or a history of cardiovascular disease: the Hoorn Study. Diabetes Care 2001;24:1793-8.

12. Maser RE, Mitchell BD, Vinik Al, et al. The association between cardiovascular autonomic neuropathy and mortality in individuals with diabetes: a meta-analysis. Diabetes Care 2003;26:1895-901.

13. Vinik Al, Maser RE, Mitchell BD, et al. Diabetic autonomic neuropathy. Diabetes Care 2003;26:1553-79.

14. Spallone V, Ziegler D, Freeman R, et al. Cardiovascular autonomic neuropathy in diabetes: clinical impact, assessment, diagnosis, and management. Diabetes Metab Res Rev 2011;27:639-53.

15. Siddig A, Hussein A. Diabetic cardiac autonomic neuropathy at Shaab Teaching Hospital among patients with coronary artery disease, unpublished manuscript, 2014.

16. Higgins JPT, Green S, eds. Cochrane handbook for systematic reviews of interventions version 5.1.0 [updated March 2011]. The Cochrane Collaboration, 2011.

17. Jaiswal M, Urbina EM, Wadwa RP, et al. Reduced heart rate variability among youth with type 1 diabetes: the SEARCH CVD study. Diabetes Care 2013;36:157-62.

18. Ziegler D, Behler M, Schroers-Teuber M, et al. Near-normoglycaemia and development of neuropathy: a 24-year prospective study from diagnosis of type 1 diabetes. BMJ Open 2015;5:e006559.

19. Pop-Busui R, Low PA, Waberski BH, et al. Effects of prior intensive insulin therapy on cardiac autonomic nervous system function in type 1 diabetes mellitus: the Diabetes Control and Complications Trial/Epidemiology of Diabetes Interventions and Complications study (DCCT/EDIC). Circulation 2009;119:2886-93.

20. Abd El Dayem SM, Battah AA, Soliman RA. Natural progression of cardiac autonomic neuropathy in patients with type 1 diabetes: a four-year follow-up study. Anadolu Kardiyol Derg 2011;11:224-31.

21. Mogensen UM, Jensen T, Kober L, et al. Cardiovascular autonomic neuropathy and subclinical cardiovascular disease in normoalbuminuric type 1 diabetic patients. Diabetes 2012;61:1822-30

22. Orlov S, Cherney DZ, Pop-Busui R, et al. Cardiac autonomic neuropathy and early progressive renal decline in patients with nonmacroalbuminuric type 1 diabetes. Clin J Am Soc Nephrol 2015;10:1136-44.

23. Witte DR, Tesfaye S, Chaturvedi N, et al. Risk factors for cardiac autonomic neuropathy in type 1 diabetes mellitus. Diabetologia 2005;48:164-71. 